\title{
SCIDoc

\section{Experience of Peripheral Arterial Disease Surgery with Tumescent Local Anesthesia}

\author{
Research Article
}

\author{
Ozyaprak B ${ }^{1 *}$, Kahraman $N^{2}$, Erkan $\mathrm{G}^{3}$, Eroglu $\mathrm{A}^{4}$
}

${ }^{1}$ Department of Anesthesiology and Reanimation, Bursa Yüksek Ihtisas Training and Research Hospital, Health Sciences University, Bursa, Turkey. ${ }^{2}$ Bursa Yüksek Ihtisas Training and Research Hospital, Health Sciences University Cardiovasculary Surgery Department, Cardiovascular Surgeon, Bursa, Turkey.

${ }^{3}$ Department of Anesthesiology and Reanimation, Ahi Evren Training and Research Hospital, Health Sciences University, Trabzon, Turkey.

${ }^{4}$ Department of Anesthesiology and Reanimation, Karadeniz Technical University, Trabzon, Turkey.

\section{Abstract}

Background: Peripheral arterial disease (PAD) is a chronic process of atherosclerosis, diabetes mellitus, hyperlipidemia, smoking, hypertension, stress and genetics. The most important symptom is ischemic claudication. General anesthesia, spinal anesthesia, local anesthesia can be performed in the surgical treatment of this disease. However, in cases where general and spinal anesthesia is very risky depending on the patient, it may be used in tumescent local anesthesia if surgery is to be performed in a large area. We retrospectively evaluated the cases in which we performed surgery for peripheral arteries with tumescent local anesthesia.

Methods: Between January 2014 and September 2018, peripheral arterial patients operated with tumescent local anesthesia were evaluated retrospectively in a Training and Research Hospital. Tumescent local anesthesia can be applied to patients at risk and when we did not prefer other anesthesia applications.

The research was performed on the patient files and the hospital information system.

Results: A total of 11 patients underwent surgical treatment for peripheral arterial disease with tumescent local anesthesia. Femoro-popliteal bypass was used in five patients, axillo-femoral bypass in two patients, axillo bifemoral bypass in one patient, femoro-femoral bypass in one patient and axillo-axillary bypass in one patient. The anesthesia duration was $128.82 \pm 10.25$ and the operation duration was $107.91 \pm 13.84$ minutes. Perioperativly hemodynamic and general status of the patients were good with tumescent local anesthesia.

Conclusion: Tumescent local anesthesia is an alternative form of application for general and spinal anesthesia patients who are too subtle and have low extremity ischemia. Close anesthesia follow-up and, if necessary, with sedoaneljezi support can be operated comfortably in patients with high operation risk.

Keywords: Tumescent Local Anesthesia; Peripheral Arterial Disease; Vascular Surgery.

\section{Introduction}

Peripheral arterial disease is a chronic disease which develops on the background of atherosclerosis and is obstructed by the occlusion of the arteries in the periphery except the coronary artery system [1]. The etiology of this disease, which is increasing in frequency in the world and in our country, includes reasons such as diabetes mellitus, hyperlipidemia, smoking, hypertension, stress and genetics [2]. Definition of PAD; Although it covers the upper extremity, extracranial carotid arteries, renal and visceral system, it has been used in practice for obstruction of the lower extremity arteries [1]. The most common and the earliest symptom in the asymptomatic disease is intermittent claudication with pain in the legs while walking [3]. Ulceration and gangrene are the most serious finding in the affected extremity. The most important finding used in clinical diagnosis is the measurement of ankle brachial index. The measurement of ankle-brachial index is less than or equal to 0.9 is diagnostic for $\operatorname{PAD}[2,3]$.

Since PAD is a systemic disease caused by atherosclerosis, the

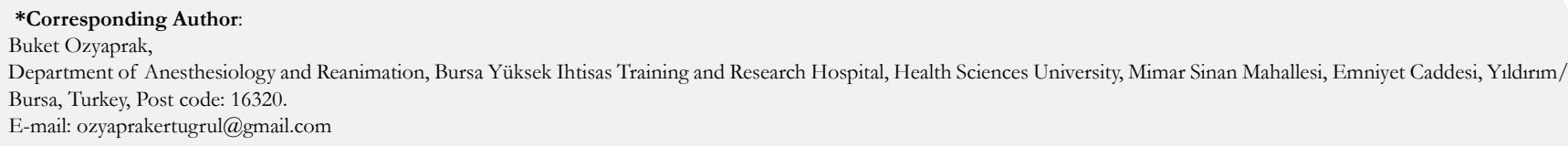

Copyright: Ozyaprak $\mathbf{B}^{\circ}$ 2019. This is an open-access article distributed under the terms of the Creative Commons Attribution License, which permits unrestricted use, distribution and reproduction in any medium, provided the original author and source are credited. 
presence of coronary artery disease, congestive heart failure and cerebrovascular disease should be considered in the patient. This also negatively affects mortality and morbidity [1, 3]. Diabetes mellitus, hypertension and chronic obstructive pulmonary diseases that may occur due to smoking and smoking increase the risk of anesthesia management in possible surgical treatments. One of the general and regional anesthesia options can be applied in the surgical treatment of PAD. However, TLA can be performed in patients with high risk of general and regional anesthesia. TLA may be a good choice, especially if a large surgical area is to be treated.

In our study; We aimed to share our experiences retrospectively by examining cases with PAD and operated with TLA due to high surgical risk.

\section{Material and Method}

Local ethics committee approval was obtained for this study. Between January 2014 and September 2018, peripheral arterial patients operated with TLA were reviewed retrospectively. Patient files and hospital information system were used for the study.

Patients' demographic data, comorbidities, American Society of Anesthesiologists (ASA) score, New York Heart Association (NYHA) functional classification, ejection fraction, anestesia and operation durations, operation type, preoperative, intraoperative and postoperative hemodynamic findings (mean arterial pressure, pulse), postoperative complications, intensive care unit (ICU) stay time and postoperative hospitalization period were examined.

TLA solution was prepared with $500 \mathrm{~mL}$ of saline, $25 \mathrm{~mL}$ of $2 \%$ lidocaine, $10 \mathrm{~mL}$ of $8.4 \%$ sodium bicarbonate and $1 \mathrm{~mL}$ of 1: 1000 epinephrine. The surgical area to be applied to TLA was marked with permanent pen before the procedure. A maximum of $500 \mathrm{ml}$ of the prepared solution was used. In addition to TLA, sedoanalgesia was performed to patients who were agitated and noncompliant.

TLA injection was applied to the surgical field by Doppler ultrasonography (DUS).

\section{The Inclusion Criteria were}

Ejection Fraction $(\mathrm{EF})<25$, NYHA functional class 3 and 4, ASA score $>3$, severe chronic obstructive pulmonary disease, history of cerebrovascular disease, diabetes mellitus, chronic renal failure, severe hepatic impairment, multiorgan dysfunction, patients with previous abdominal operation history.

\section{The Exclusion Criteria were}

$\mathrm{EF}>25$, NYHA 1 and 2, ASA score $<3$, patients with no contraindications to spinal and general anesthesia.

\section{Anesthesia Procedure}

Premedication was not performed except for cardiac drugs in the morning. SpO2 monitarization was performed with pulse oximetry and electrocardiographic monitoring in leads D2 and V5 in the operating room. Peripheral venous vascular access was opened to the patients with a 16 or 18 gauge cannula. Systemic arterial pressure monitoring was performed invasively by radial artery cannulation from non-dominant arm.

If necessary, sedoanalgesia in addition to TLA, intravenous 0.5 $1 \mathrm{mg} / \mathrm{kg}$ ketamine according to the hemodynamic status of the patient (Ketalar ${ }^{\circledR}$ Phizer drugs LTD. Sti., Turkey) and intravenous 0.05-0.1 mgr $/ \mathrm{kg}$ Midazolam (Dormicum ${ }^{\circledR}$ Deva Holding A.Ş., Turkey. If the patient developed respiratory depression, the patient was ventilated with a mask until his spontaneous breathing returned. After surgery, the patient was transferred to the cardiovascular surgery intensive care unit.

\section{Surgical Procedure}

Axillofemoral bypass/axillobifemoral bypass: Preoperatively, TLA injection was performed on the surgical field with DUS. A short incision was made in 1/3 middle section of the clavicle. Pectoralis major muscle fibers were separated. Axillary artery was returned with nylon tape. If we want to widen the surgical field, we can cut the pectoralis minor muscle. A $6 \mathrm{~mm}$ (polytetrafluoroethylene) PTFE graft was used. The proximal anastomosis was performed with $5 / 0$ prolene suture to the axillary artery. A subcutan tunnel was created from the lateral chest wall and the lateral side of the abdomen, starting from the middle axillary line with the long artery tunneling agent, and from the line marked with a permanent pen before the surgical procedure (Figure 1). The distal anastomosis was performed with $6 / 0$ prolene suture to the femoral artery.

Figure 1. Marking the surgical area with a permanent pen before surgery in the axillo femoral bypass patient with TLA.

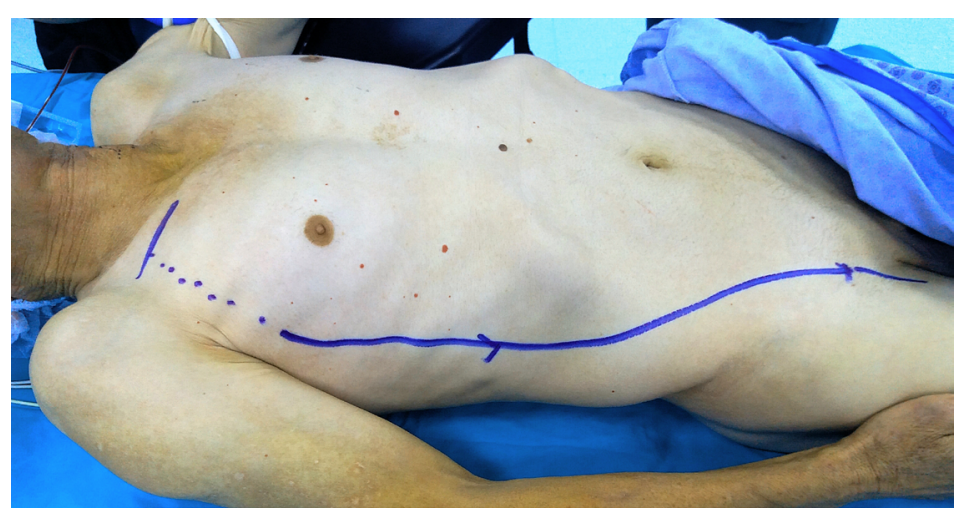


Axillo-axillary bypass: TLA with DUS was applied to the surgical area which was preoperatively marked with permanent pen. A short skin incision was made in 1/3 middle section of the clavicle. Pectoralis major muscle fibers were separated. Axillary artery was returned with nylon tape. If necessary, the pectoralis minor muscle may be cut to extend the surgical field of view. Proximal anastomosis was performed with $5 / 0$ prolene suture using a 6 $\mathrm{mm}$ PTFE graft. The axillary artery against the graft was passed in front of the sternum as a subcutane. Distal anastomosis was performed with $5 / 0$ prolene suture.

Femoro-popliteal bypass: TLA with DUS was applied to the surgical area which was preoperatively marked with permanent pen (Figure 2). Femoral artery and popliteal artery exploration were performed. A $6 \mathrm{~mm}$ PTFE graft was inserted from the femoral artery to the popliteal artery, passing under the musculus sartorius with the long vessel tunnel opener. Distal and proximal anastomosis were performed with $6 / 0$ prolene sutures.
Femoro-femoral bypass: TLA with DUS was applied to the surgical area which was preoperatively marked with permanent pen. Both femoral veins were explored. A subcutan tunnel was created with a long vessel tunneling from the anterior abdominal wall. Bypass procedure was performed with $6 \mathrm{~mm}$ PTFE graft and 6/0 prolene suture.

\section{Results}

A total of 11 patients ( 8 males, 3 females) underwent surgical treatment with TLA. The mean age of our patients was $68.27 \pm$ 13.24 (Table 1). All patients had ejection fraction less than $25 \%$ and decompensated heart failure. In addition, all patients had hypertension, hyperlipidemia, diabetes mellitus and chronic obstructive pulmonary disease. As an additional disease, one of our cases had cerebrovascular disease and three had morbid obesity. Demographic data and preoperative risk factors of our patients are summarized in Table 1.

Figure 2. Marking of the surgical area with a permanent pen before surgery in the case of femoro-popliteal bypass in TLA.

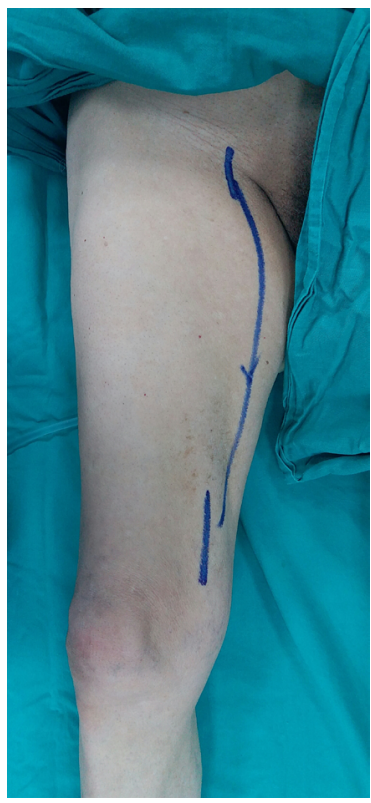

Table 1. Demographic Data and Preoperative Risk Factors.

\begin{tabular}{|c|c|c|}
\hline & Number (n) & Percent (\%) \\
\hline Female & 3 & 27.28 \\
\hline Male & 8 & 72.72 \\
\hline ASA 4 & 11 & 100 \\
\hline NHYA 3 & 5 & 45.45 \\
\hline NHYA 4 & 6 & 54.55 \\
\hline Chronic Obstructive Pulmonary Disease & 11 & 9.09 \\
\hline Diabetes Mellitus & 11 & 100 \\
\hline Hyperlipidemia & 11 & 100 \\
\hline Hypertension & 11 & 100 \\
\hline Cerebrovascular Event & 1 & 9.09 \\
\hline Morbit Obesity & 3 & 27.28 \\
\hline Mean Age(Years) \pm SD & $68.27 \pm 13.24$ & \\
\hline
\end{tabular}


The anesthesia duration was $128.82 \pm 10.25$ and the operation duration was $107.91 \pm 13.84$ minutes. Preoperative, intraoperative and postoperative mean arterial pressures were as follows; $99.55 \pm 15.48,75.18 \pm 10.99,79.36 \pm 9.87 \mathrm{~mm} / \mathrm{Hg}$ (Table 2). The preoperative, intraoperative and postoperative pulse values of the patients were $70.45 \pm 11.36,69.27 \pm 11.14,70.55 \pm 11.04$ beat/ minute, respectively (Table 2 ).
Five patients had femoro-popliteal bypass, two patients had axillo femoral bypass, 1 patient had axillo bifemoral bypass, two patients underwent femoro-femoral bypass and one patient axillo-axillary bypass. Early postoperative complications are summarized in $\mathrm{Ta}-$ ble 3. One of our patients died because of multi-organ failure.

Table 2. Anestesia and Operation Durations, Hemodynamic Findings.

\begin{tabular}{|c|c|c|}
\hline & Mean umber $(\mathbf{n})$ & Standard Deviation $(\mathbf{\pm})$ \\
\hline Anestesia Duration (minute) & 128.82 & 10.25 \\
\hline Operation Duration (minute) & 107.91 & 13.84 \\
\hline Preoperative Pulse (beat/minute) & 70.45 & 11.36 \\
\hline Intraoperative Pulse (beat/minute) & 69.27 & 11.14 \\
\hline Postoperative Pulse (beat/minute) & 70.55 & 11.04 \\
\hline Preoperative Mean Arterial Pressure $(\mathrm{mm} / \mathrm{Hg})$ & 99.55 & 15.48 \\
\hline Intraoperative Mean Arterial Pressure $(\mathrm{mm} / \mathrm{Hg})$ & 75.18 & 10.99 \\
\hline Postoperative Mean Arterial Pressure $(\mathrm{mm} / \mathrm{Hg})$ & 79.36 & 9.87 \\
\hline
\end{tabular}

Table 3. Types of Surgical Operations and Postoperative Findings.

\begin{tabular}{|c|c|c|c|c|}
\hline & $\begin{array}{c}\text { Operation Type } \\
\text { umber((n) }\end{array}$ & $\begin{array}{c}\text { Intensive Care } \\
\text { Period/h }\end{array}$ & $\begin{array}{c}\text { Hospitalization } \\
\text { Time/day }\end{array}$ & $\begin{array}{c}\text { Postoperative } \\
\text { Complications }\end{array}$ \\
\hline CASE 1 & Axillo-femoral bypass & 20 & 3 & - \\
\hline CASE 2 & Femoro-popliteal bypass & 14 & 3 & - \\
\hline CASE 3 & Femoro-popliteal bypass & 21 & 4 & - \\
\hline CASE 4 & Axillo-Bifemoral bypass & 24 & 6 & $\begin{array}{c}\text { Superficial skin } \\
\text { infection }\end{array}$ \\
\hline CASE 5 & Femoro-popliteal bypass & 15 & 4 & - \\
\hline CASE 6 & Axillo-axillary bypass & 25 & 4 & - \\
\hline CASE 7 & Axillo-femoral bypass & 20 & 3 & - \\
\hline CASE 8 & Femoro-femoral bypass & 46 & 2 & Exitus \\
\hline CASE 9 & Femoro-popliteal bypass & 22 & 5 & - \\
\hline CASE 10 & Femoro-femoral bypas & 18 & 4 & - \\
\hline CASE 11 & Femoro-popliteal bypass & 18 & 7 & Cerebrovascular \\
Event
\end{tabular}

\section{Discussion}

PAD is a disease with high mortality and morbidity. Treatment of the disease; It is very important to take measures for preventable predisposing factors. In the etiology of the disease; Smoking, hypertension, hyperlipidemia, diabetes mellitus are important risk factors [2]. All patients operated with TLA had diabetes mellitus, smoking, hypertension and hyperlipidemia. Male gender and older age are risk factors for PAD [2]. The mean age of our cases was 68.27 (46-84), and 8 of the 11 patients operated were men.

PAD may also affect coronary, cerebral and renal vessels as it develops from atherosclerosis. These patients are also at high risk for myocardial infarction, cerebrovascular disease and death [4]. In particular, a history of myocardial infarction and chronic obstructive pulmonary diseases; predisposing factors for heart failure. All of our patients had long-lasting smoking and chronic obstructive pulmonary disease. When we evaluated our cases in terms of heart failure, all of our patients had EF values less than $25 \%$.

Heart failure and chronic obstructive pulmonary disease are factors that increase the risk in terms of anesthesia. According to the American Society of Anesthesiologist classification; The patient with ASA score of four is a person who has completely lost strength and has a life threatening condition (such as shock, decompensated heart failure or respiratory system disease, kidney and liver failure) [5]. The ASA score of all our patients who underwent surgery for their PAD with TLA was four.

In addition, anticoagulant therapy that cannot be applied to peripheral artery patients before and after the operation is also contraindication for regional anesthesia due to possible coagulopathy and bleeding diathesis [6]. The use of anticoagulants in our pa- 
tients continued until the day of operation due to cric ischemia.

A previous lumbar surgery is a relative contraindication for spinal anesthesia [6]. Two of our cases had a previous history of lumbar surgery.

TLA; It can be used in cases where general and regional anesthesia cannot be applied. If necessary, it can be supplemented with sedoanalgesia and a good alternative to other anesthetic methods. TLA is injected with the local anesthetic drug by dilution with saline and then injected into the region under DUS guidance.7 The most important thing to consider in preventing the complication of methemoglobinemia due to overdose local anesthesia is to determine the maximum local anesthetic dose to be given to the patient's weight [7]. In our cases, the local anesthesia dose was calculated according to the weight of the patient and no complication of methemoglobinemia was found.

TLA was initially used widely in Liposuction [8-11]. TLA is widely used in endovenous thermal ablation and catheter-mediated foam sclerotherapy for reducing venous diameter, providing anesthesia and protection of peri-saphenous tissues in the treatment of lower extremity varices [12-15].

Ersoy et al., 12 patients with the diagnosis of carpal tunnel syndrome have shared their experiences in their work [16].

They argued that this surgical procedure with TLA has advantages such as being more practical, short-term and significantly shortening the length of hospital stay. In our patients with many additional diseases, the duration of hospitalization was similar to patients undergoing spinal or general anesthesia (3-7 days).

Yilmaz et al., In a case report of patients with peripheral arterial disease, they reported that the use of TLA may be an alternative to other anesthetic methods in a patient undergoing extra-anatomical bypass [17]. When we evaluated the patients in terms of operation results, postoperative mortality occurred in one patient. In a case report published by Mizukami et al. They underwent revascularization of a patient with coronary subclavian steal syndrome who underwent TLA [18]. In a study by Martin et al., The operative mortality of the axillofemoral bypass was $4.9 \%$, whereas the operative mortality of the aortic femoral bypass was $12.1 \%$ [19].

There are some limitatons of our study. For example sample size was small and the design of our study was retrospectivly. We need further prospective clinical studies in this area.

\section{Conclusion}

Early diagnosis is important because of the risks caused by PAD. In addition, the treatment should be done immediately is a disease. Since it is more comfortable for the patient and surgeon in the surgical treatment, general and spinal anesthesia is applied as the first choice. But the operation process in the presence of comorbid diseases and the risk of high anesthesia can be an alternative anesthetic method of TLA, which should be followed more closely. To the best our knowledge, we have not seen much literature on the use of TLA in peripheral arterial patients in the literature scans. When we evaluated the results of high-risk patients in terms of the operation we had to operate with TLA. We believe that many vascular surgical procedures can be performed successfully with the help of experienced anesthesiologist and vascular surgeon.

\section{References}

[1]. Balkanay OO, Ömeroglu NS. Approach to Peripheral Artery Disease in the Elderly. Turk Kardiyol Dern Ars. 2017 Sep;45(Suppl 5):96-101. PubMed PMID: 28976393

[2]. Karabay Ö, Karacelik M, Yilik L, Tekin N, Iriz AB, Kumdereli, S, et al. Ischemic Peripheral Arterial Disease: A Screening. Turk Thorac Cardiovasc Surg. 2012;20(3):450-457.

[3]. Sadikoglu G, Ozcakir A, Uncu Y, Yildiz Y. Risk factors and clinical findings of peripheral arterial diseases. J of Uludag University Faculty of Med. 2002;28(3):77-80

[4]. Bozoglan O. Diabetes Mellitus and Peripheral Artery Disease. Kahramanmaras Sutcu Imam University Medical Faculty Journal 2015;10 (2):64-67.

[5]. TARD (Turkish Society of Anesthesiology and Reanimation), Preoperative Evaluation Guide. Anesthesia Practice Guidelines 2015; a, 1-24.

[6]. TARD (Turkish Society of Anesthesiology and Reanimation), Guidelines for Use of Neuroaxial Anesthesia in Caesarean Surgery. Anesthesia Practice Guidelines 2015; b, 1- 36 .

[7]. Çil B. Treatment of Lower Extremity Varicose Veins. Turkish Radiology Seminars 2015; 3:316-327. PubMed PMID: 28976393

[8]. Klein JA. Tumescent technique for local anesthesia improves safety in largevolume lipoauction. Plast Reconstr Surg. 1993 Nov;92(6):1085-98. PubMed PMID: 8234507.

[9]. Habbema L. Safety of liposuction using exclusively tumescent local anesthesia in 3,240 consecutive cases. Dermatol Surg. 2009 Nov;35(11):1728-35. PubMed PMID: 19660028.

[10]. Hanke CW, Bernstein G, Bullock S. Safety of tumescent liposuction in 15,336 patients: National survey results. Dermatol Surg. 1995 May;21(5):459-62. PubMed PMID: 7743109.

[11]. Habbema L. Efficacy of tumescent local anesthesia with variable lidocaine concentration in 3430 consecutive cases of liposuction. J Am Acad Dermatol. 2010 Jun;62(6):988-94.PubMed PMID: 20466173.

[12]. Parsi K. Catheter-directed sclerotherapy. Phlebology. 2009 Jun;24(3):98107. PubMed PMID: 19470860.

[13]. Thibault P. Internal compression (peri-venous) following ultrasound guided sclerotherapy. Aust NZ J Phleb. 2005;9:29-32.

[14]. Deverux N, Recke AL, Westermann L, Recke A, Cahle B. Catheter-directed foam sclerotherapy of great saphenous veins in combination with pretreatment reduction of the diameter employing the principals of perivenous tumescent local anesthesia.Eur J Vasc Endovasc Surg 2014; 47:187-195. PubMed PMID: 24268395.

[15]. Cavezzi A, Mosti G, Di Paolo S, Tessari L, Campana F, Urso SU. Ultrasound-guided perisaphenous tumescence infiltration improves the results of long-term catheter foam sclerotherapy combined with phlebectomy of the varicose tributaries. Veins and Lymphatics 2015; 4:18-23.

[16]. Ersoy B, Sönmez A, Numanoglu A. Nonlinear Carpal Tunnel Liberalization Under Tumulus Anesthesia. Turkish Journal of Plastic Reconstructive and Aesthetic Surgery 2010;18 (1): 11- 17.

[17]. Yilmaz S, Barıs O, Yalcınkaya A, Yilmaz BK. Extra-Anatomical By-Pass with Tachyrene Anesthesia: Case Report. Kocaeli Medikal J 2016; 5 (2): 46-50.

[18]. Mizukami T, Hamamoto M. Tumescent local anesthesia for a revascularization of a coronary subclavian steal syndrome. Ann Thorac Cardiovasc Surg. 2007 Oct;13(5):352-4. PubMed PMID: 17954996.

[19]. Martin D, Katz SG. Axillofemoral bypass for aortoiliac occlusive disease. Am J Surg. 2000 Aug;180(2):100-3. PubMed PMID: 11044521. 